more variables, occurring in the WHERE clause, that express the grouping criteria; and (d) an optional FILTER clause.

The semantics of a query with aggregate functions consists in adding to the regular variable bindings computed by the wHERE clause some new bindings, one for each of the new variables introduced by the AGGREGATE clauses. The solution constructed in this way may be further filtered by the FILTER clause. The evaluations of aggregate functions are all independent from each other and take place after the computation of the bindings provided by the wHERE clause.

\section{EXAMPLES OF C-SPARQL}

A simple Query with Aggregation - Aggregation is orthogonal w.r.t. the other extensions, so we start with a query having aggregates but no streams. It counts the number of sensors placed in every street and returns those with more than 5 sensors. The query is not continuous and requires no registration.

PREFIX c: 〈http://linkedurbandata.org/city\#〉 SELECT DISTINCT ?street ? sensors

WHERE \{ ?sensor c:placedIn ?street. \}

AGGREGATE $\{$ ( ?sensors, COUNT, \{? street\}) FILTER (?sensors > 5)\}

The query is executed by first extracting all pairs of bindings of sensors with their street, then the number of sensors in each street is counted into the new variable sensors and each resulting pair is extended into a triple, then the triples which satisfy the filter predicate are selected, and finally distinct pairs of street and sensor numbers are projected.

A simple Query over a Stream - A classic example in Urban Computing is counting the cars enter the city center passing through tollgates. The next query counts how many cars went through each tollgate in the last 10 minutes, sliding the window every minute.

REGISTER QUERY CarsEnteringCityCenterPerTollgate AS

PREFIX $t:<$ http://linkedurbandata.org/traffic\#>

SELECT DISTINCT ?tollgate ?passages

FROM STREAM <WWW.uc.eu/tollgates.trdf> [RANGE 10 MIN STEP 1 MIN] WHERE \{ ?tollgate t:registers ?car.

AGGREGATE \{(?passages, COUNT, \{?tollgate\})\}

First, all pairs of bindings of tollgates with the car they register are extracted from the current window, then the number of cars is counted into the new variable passages for each tollgate (and each resulting pair is extended into a triple), and finally the result is projected as distinct pairs of tollgate and passages. Note that at every new minute new triples enter into the window and old triples exit, and the query result does not change during the slide interval; it changes only at every slide change (i.e., at every minute).

In this stream, as in all the streams that we will use in the examples of this paper, the predicate of the triple (e.g. $t$ :register) is fixed while the subject and object part of the triple (e.g., ?tollgate and ?car) are variable. Thus, a physical source for this stream will have items consisting of pairs of values. This arrangement is coherent with RDF repositories whose predicates are taken from a small vocabulary constituting a sort of schema, but C-SPARQL makes no assumption on variable bindings of its stream triples.

Combining Static and Streaming Knowledge - A more complex example counts the number of car entering the city center from each district. The RDF repository stores (a) which districts a city is divided in, (b) which streets belong to each district, and (c) which street each tollgate is placed in. The window is set to 30 minutes and slides every 5 minutes. For brevity, the declaration of prefixes $c$ : and $t$ : will be omitted in the next examples.
REGISTER QUERY CarsEnteringCityCenterPerDistrict AS SELECT DISTINCT ?district ?passages

EROM STREAM <WWW.uc.eu/tollgates.trdf> [RANGE 30 MIN STEP 5 MIN]

WHERE $\{$ ?toll t:registers ?car. ?toll c:placedin ?street. ?district $c$ :contains ?street.$\}$

AGGREGATE \{ (?passages, COUNT, \{?district \}) \}

As in the previous query, all pairs of bindings of tollgates with the cars are extracted. Also, a graph pattern also extracts the pair of bindings of tollgates with the district they are in. Here the cars are counted based on the district.

Streaming the Results of a Query - Continuous queries renew their output at each query execution; such output could be periodically transferred to another system for further analysis (e.g., to plot the traffic as a function of time). In addition, C-SPARQL allows the construction of new RDF data streams, by supporting the possibility to register CONSTRUCT and DESCRIBE queries. We can register the previous query to generate a stream of RDF triples:

REGISTER STREAM CarsEnteringCityCenterPerDistrict AS CONSTRUCT \{?district t:has-entering-cars ?passages\}.

FROM STREAM <WWW.uc.eu/tollgates,trdf> [RANGE 30 MIN STEP 5 MIN]

WHERE \{ ?toll t:registers ?car. ?toll c:placedIn ?street. ?district c:contains ?street. ?

AGGREGATE \{ (?passages, COUNT, \{?district\}) \}

Every query execution may produce from a minimum of one triple to a maximum of an entire graph. In the former case, a different timestamp is assigned to every triple; in the latter case, the same timestamp is assigned to all the triples of the graph. In both cases, timestamps are systemgenerated in monotonic order.

Combining Multiple Streams - We now also consider traffic control cameras registering cars at traffic lights, originating a different stream. The next query finds the streets that have been over $80 \%$ of their capacity in the last 5 minutes and shows the number of cars (cars seen by cameras and passing through tolls are summed up).

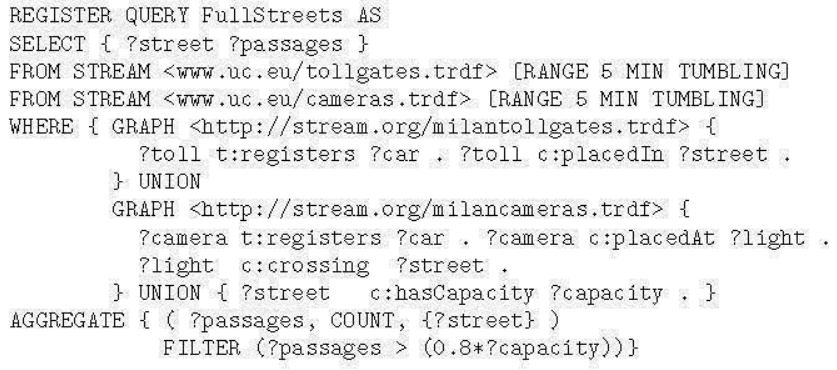

Here, the bindings over the different graphs are combined following the semantics of the UNION pattern evaluation in SPARQL, and it becomes possible to count in the new variable passages the cars registered either by the tollgates or by the cameras in each street.

\section{Acknowledgement}

This work is supported by the FP7-215535 integrated project (LarKC) funded by the E.U. Dr. Grossniklaus's work is carried out under SNF grant number PBEZ2-121230. We acknowledge Ioana Manolescu for her contributions to the initial discussions on the potential impact of RDF streams on several use cases.

\section{REFERENCES}

[1] A. Arasu, S. Babu, and J. Widom. The CQL Continuous Query Language: Semantic Foundations and Query Execution. The VLDB Journal, 15(2):121-142, 2006.

[2] E. Prud'hommeaux and A. Seaborne. SPARQL Query Language for RDF Grammar. http: //www. w3. org/TR/rdf-sparql-query/\#sparqlGrammar. 